\title{
New insights on homogenization for hexagonal-shaped composites as Cosserat continua
}

\author{
Marco Colatosti $\cdot$ Nicholas Fantuzzi $\cdot$ Patrizia Trovalusci $(\mathbb{D}) \cdot$ Renato Masiani
}

Received: 15 January 2021 / Accepted: 4 April 2021 / Published online: 30 April 2021

(C) The Author(s) 2021

\begin{abstract}
In this work, particle composite materials with different kind of microstructures are analyzed. Such materials are described as made of rigid particles and elastic interfaces. Rigid particles of arbitrary hexagonal shape are considered and their geometry is described by a limited set of parameters. Three different textures are analyzed and static analyses are performed for a comparison among the solutions of discrete, micropolar (Cosserat) and classical models. In particular, the displacements of the discrete model are compared to the displacement fields of equivalent micropolar and classical continua realized through a homogenization technique, starting from the representative elementary volume detected with a numeric approach. The performed analyses show the effectiveness of adopting the micropolar continuum theory for describing such materials.
\end{abstract}

M. Colatosti · P. Trovalusci $(\bowtie) \cdot$ R. Masiani

DISG Department, Sapienza University of Rome, Via A. Gramsci 53, 00197 Rome, Italy

e-mail: patrizia.trovalusci@uniroma1.it

M. Colatosti

e-mail: marco.colatosti@uniroma1.it

R. Masiani

e-mail: renato.masiani@uniroma1.it

N. Fantuzzi

DICAM Department, University of Bologna, Viale del

Risorgimento 2, 40136 Bologna, Italy

e-mail: nicholas.fantuzzi@unibo.it
Keywords Composite materials - Cosserat theory Homogenization · RVE · Hexagonal shaped particles . Finite element method

\section{Introduction}

Composite materials can be investigated by directly describing their constituents in a micromechanical discrete model or by homogenizing them as equivalent continua. In the former case, for example to study heterogeneous materials such as polycrystalline materials, jointed rock systems, and block masonry with periodic microstructures, the model involves a system with a large numbers degrees of freedom and as a consequence the computational costs is very high [1-4]; such cost increases by reducing the material scale [5]. In the latter case, by using homogenization techniques, the numerical analyses are faster and more efficient but it is fundamental to define equivalent continua that properly takes into account the influence of the microstructure on the macro-scale behaviour with particular reference to shape, size and texture of elements [6] and among them focus on those techniques developed for deriving scale-dependent models [7-12]. When classical kinematics is enriched with extra degrees of freedom for instance, homogenization procedures have been shown to provide more reliable 
models than in the case of classical, local, continua, even in the case of higher order continua [10, 13-15].

A model can be defined as non-local due to the presence of both internal length scales and dispersion properties, revealing in some cases the existence of an underlying microstructure which inevitably affects the macroscopic mechanical properties $[11,16]$. In such non-local models the governing equations contain one or more parameters that take in account material internal lengths and present dispersion properties in wave propagation [17-22]. Within these models, micromorphic continua, in particular continua with rigid local structure (micropolar or Cosserat) [23-27], show the advantages of a coarse-scale field description while keeping the memory of the fine organization of the material and in particular of material internal length. These models, which present additional degrees of freedom, can be classified as 'implicit/ weak' non-local models [11, 28, 29]. The literature shows that they have been satisfactorily applied to various composites [10, 13, 30-40]. Different works moreover, [41-46] proposed multiscale computational homogenization with 'explicit' non-local or higher order deformation gradient theories in composite materials [47-51]. In particular non-local models with gradient elasticity has been used to study one-dimensional structures such as beams and rods [52, 53].

The selection of proper non-local continuum theories which retain memory of the fine internal structure of the material, shape, size and texture, is then a fundamental point in the macroscopic description of composite materials. Among various homogenization techniques, the development of methods and conceptual guidelines for obtaining continuous field descriptions by linking discrete and continuum solid mechanics have been successfully employed material science. In this work, a micromechanical approach based on the scale dependent discrete-continuum homogenization procedure defined in [10, 35] for particle composite materials, addressed to derive the macroscopic mechanical properties of a micropolar continuum, is adopted. The micropolar theory, beside the standard displacement, introduces the rotation of the material point, termed microrotation, to be distinguished with the macrorotation (local rigid rotation). When the relative rotation, i.e. the difference between micro and macrorotation, is null the micropolar continuum, under some other dynamical costraints, behaves as a couple stress continuum [13]. As the effects of the relative rotation are expected to be prominent for strongly anisotropic particle composites, as widely investigated in [14, 15] for masonrylike materials, we consider here the full (unconstrained) micropolar theory.

In order to highlight the advantages of micropolar elasticity description, the numerical solutions of equivalent micropolar and classical continua, obtained by putting some internal constraint to the micropolar model, are compared to the one of a discrete model assumed as benchmark solution. The discrete numerical solution has been obtained using the ABAQUS software in which the single blocks are rigid and elasticity is concentrated at the interfaces through elastic linear springs (joints) while for the continuum, classical and micropolar, solution, an in-house FEM code has been performed [37, 54]. Moreover, an investigation is proposed in order to detect the representative volume element for hexagonal shaped composites. This geometry is typical for some polycrystals with thin interfaces such as Aluminia $\left(\mathrm{Al}_{2} \mathrm{O}_{3}\right)$, Zirconia $\left(\mathrm{ZrO}_{2}\right)$, Zinc Ozide $(\mathrm{ZnO})$ or TungstenCarbide (WC). Considering a generic tile (e.g., orientation of the interfaces and internal angles of the hexagonal geometry) different material symmetries can be detected [55]. The hexagonal shaped composites are investigated starting from the previous works [37, 39] and in particular considering three microstructure geometries [40].

In this paper, at first Cosserat theoretical aspects are briefly presented (Sect. 2) and then the geometries of microstructured composites are be introduced, in particular by studying the unite cell to detect the RVE necessary for the homogenization procedure (Sect. 3). Later the numerical implementation of the continuum model is shown (Sect. 4), the discrete model assumed as benchmark solution is properly described and finally a static analyses comparison among the discrete system, the micropolar and the classical continuum for a $2 \mathrm{D}$ panel is reported (Sect. $6)$.

\section{Cosserat theoretical framework}

The Cosserat model can be considered equivalent to an assembly of rigid particles undergoing homogeneous displacements and rotations which interact with each other via forces and couples. Therefore, it is 
suitable for simulating the mechanical response of granular, masonry-like and composite materials with either periodic or random microstructure $[10,33,36]$.

For the present study a 2D rectangular panel with three different hexagonal microstructures is considered. In a linearized 2D framework there are two displacements and one rotation component, so that the generalized displacements $\quad \underline{u}^{T}=\left[\begin{array}{lll}u_{1} & u_{2} & \omega\end{array}\right]$ applies. The strain displacement vector is $\underline{\varepsilon}^{T}=\left[\begin{array}{llllll}\varepsilon_{11} & \varepsilon_{22} & \varepsilon_{12} & \varepsilon_{21} & k_{1} & k_{2}\end{array}\right], \quad$ where $\varepsilon_{11}, \varepsilon_{22}, \varepsilon_{12}, \varepsilon_{21}$ are the in-plane normal and shear strains and $k_{1}, k_{2}$ are the micropolar curvatures. Note that the strain components are not symmetric $\varepsilon_{12} \neq \varepsilon_{21}$. Moreover, the microrotation $\omega$ is different from the local rigid rotation (macrorotation) $\theta$, defined as the skew-symmetric part of the gradient of displacement $\theta=\frac{1}{2}\left(\frac{\partial u_{2}}{\partial x_{1}}-\frac{\partial u_{1}}{\partial x_{2}}\right)$ and the difference between the two rotations $\theta-\omega$ defines the strain measure of the relative rotation, corresponding to the skew-symmetric part of the strain. When the relative rotation equals zero $\theta=\omega$ then $\varepsilon_{12}=\varepsilon_{21}=\frac{\partial u_{2}}{\partial x_{1}}-\frac{\partial u_{1}}{\partial x_{2}}$ as in the classical continuum, the micropolar continuum becomes a continuum with constrained rotations, that under other dynamical constrains, is a couple stress continuum $[13,56]$.

The stress vector is represented as: $\underline{\sigma}^{T}=$ $\left[\begin{array}{llllll}\sigma_{11} & \sigma_{22} & \sigma_{12} & \sigma_{21} & \mu_{1} & \mu_{2}\end{array}\right]$ where $\sigma_{i j}$ for $i, j=$ 1,2 represent the classical normal and shear stress components and $\mu_{1}, \mu_{2}$ are the microcouples. The stress components are not reciprocal, $\sigma_{12} \neq \sigma_{21}$ and the couple stress components $\mu_{1}, \mu_{2}$ have to be introduced in order to satisfy the moment equilibrium of the micropolar body.

The kinematic compatibility relations can be written as:

$\underline{\varepsilon}=\underline{D} \underline{u}$

where:

$$
\underline{D}=\left[\begin{array}{ccc}
\frac{\partial}{\partial x_{1}} & 0 & 0 \\
0 & \frac{\partial}{\partial x_{2}} & 0 \\
\frac{\partial}{\partial x_{2}} & 0 & 1 \\
0 & \frac{\partial}{\partial x_{1}} & -1 \\
0 & 0 & \frac{\partial}{\partial x_{1}} \\
0 & 0 & \frac{\partial}{\partial x_{2}}
\end{array}\right]
$$

From the virtual work principle, equilibrium equations in terms of stresses and microcouples can be carried out as $\delta U+\delta V=0$, being $\delta U$ the internal work:

$\delta U=\int_{V} \delta \underline{\varepsilon}^{T} \underline{\sigma} d V=h \int_{A} \delta \underline{u}^{T}\left(\underline{D}^{T} \underline{\sigma}\right) d A$

and $\delta V$ the potential of external loads, that in $2 \mathrm{D}$ domain takes the form:

$\delta V=-\int_{V} \delta \underline{u}^{T} \underline{f} d A-\int_{S} \delta \underline{u}^{T} \underline{p} d S$

where $f$ and $\underline{p}$ are the vectors of body forces and boundary tractions, respectively. Balance domain equations are then given by:

$\underline{D}^{T} \underline{\sigma}=\underline{f}$

and boundary tractions as:

$\underline{p}=\underline{\hat{p}}$

where $\hat{p}$ are respectively, contact forces and couples applied at the boundary.

The micropolar anisotropic constitutive equations take the form:

$\underline{\sigma}=\underline{C} \underline{\varepsilon}$

where: 


$$
\underline{C}=\left[\begin{array}{cccccc}
A_{1111} & A_{1122} & A_{1112} & A_{1121} & B_{111} & B_{112} \\
& A_{2222} & A_{2212} & A_{2221} & B_{221} & B_{222} \\
& & A_{1212} & A_{1221} & B_{121} & B_{122} \\
& & & A_{2121} & B_{211} & B_{212} \\
& & & & D_{11} & D_{12} \\
\text { sym } & & & & & D_{22}
\end{array}\right]
$$

Due to hyperelasticity, the constitutive matrix is symmetric $(\underline{C} \in S y m)$.

Accounting for the constitutive equations, the internal work can be write:

$$
\delta U=\int_{V} \delta \underline{\varepsilon}^{T} \underline{\sigma}=\int_{V} \delta_{\underline{\varepsilon}}^{T} \underline{C} \underline{\varepsilon} d V=h \int_{A}\left(\delta \underline{u}^{T} \underline{D}^{T} \underline{C} \underline{D} \underline{u}\right) d A
$$

In Sect. 4 the principle of virtual work will be adopted for the numerical implementation.

\section{Microstructure geometry}

The geometry of the microstructure is realized considering a six edges parallelogram and it is possible to give different input settings to set the shape: the main parameters are the angles $\alpha_{1}, \alpha_{2}$ and $\alpha_{3}$ and the relative length (Fig. 1), $l_{r}=A E /\left(A E+l_{1}\right)=$ $1 /(1 / \sqrt{3}+1)$ that defines the ratio between the length of the base $A E$ (or $B D$ ) and the $l_{1}$ (perpendicular distance between $A E$ and $B D$ ), $l_{1}, l_{2}$ are defined as: $l_{2}=s \cdot l_{r}$ where $s$ is the microstructure scale factor, $l_{1}=1-l_{2}[40,57]$.

Keeping constant the lengths, and varying the angles $\alpha_{2}$ and $\alpha_{3}$ it is possible to obtain different geometries; in particular for $\alpha_{2}=0$ and $\alpha_{3}=0$ the lateral edges of hexagonal shape are vertical therefore the parallelogram acquires a rectangular shape. In this paper, for the patterns studied, the angles $\alpha_{2}$ and $\alpha_{3}$ assume the values: $\alpha_{2}=\alpha_{3}=30^{\circ}$ for the regular hexagonal shape, $\alpha_{2}=\alpha_{3}=-20^{\circ}$ for the hourglass shape and $\alpha_{2}=-\alpha_{3}=-30^{\circ}$ for the skew shape. Finally, by varying the relative scale the three geometries reported in Fig. 2 are obtained by setting the scale factor $s$ : where $s=1$ for large blocks, $s=0.5$ for medium blocks and $s=0.25$ for small blocks. The ratios among the parameter $l_{2}$ of microstructure shape and the vertical side $L_{y}$ of panel analyzed in Sect. 5 are: $l_{2} / L_{y}=0.0411, l_{2} / L_{y}=0.0823 l_{2} / L_{y}=0.0205$.

\subsection{Representative volume element}

The homogenization procedure adopted for deriving the material constants of the micropolar continuum is the one defined in [10], where at the fine level the material is described as a system of rigid blocks, here named elements, interacting through elastic contact elements, here referred as joints (e.g. springs of normal stiffness $k_{11}=785 \mathrm{~N} / \mathrm{m}$ and shear stiffness $k_{22}=$ $392.5 \mathrm{~N} / \mathrm{m}$ ), the interaction is represented by forces and couples. A kinematic correspondence map between discrete and continuum fields is assumed (generalization of the Cauchy-Born rule) and an energy equivalence criterion is adopted. To apply the homogenization procedure for periodic assemblies it is necessary to firstly define the Representative Volume Element (RVE), as the elementary volume element (unit cell) made of the minimal number of elements and joints sufficient to completely define the behavior of the discontinuous and heterogeneous material. As the micropolar continuum is intrinsically


Fig. 1 Hexagonal shape pattern and single tile for the parameters: $\alpha_{1}=25^{\circ}, \alpha_{2}=45^{\circ}, \alpha_{1}=30^{\circ}$ and $l_{r}=40$ 


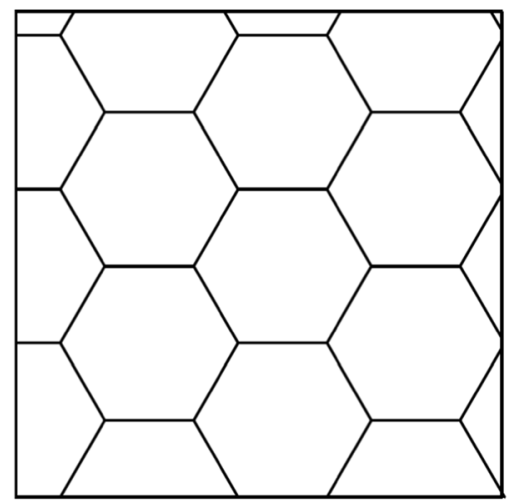

(a)

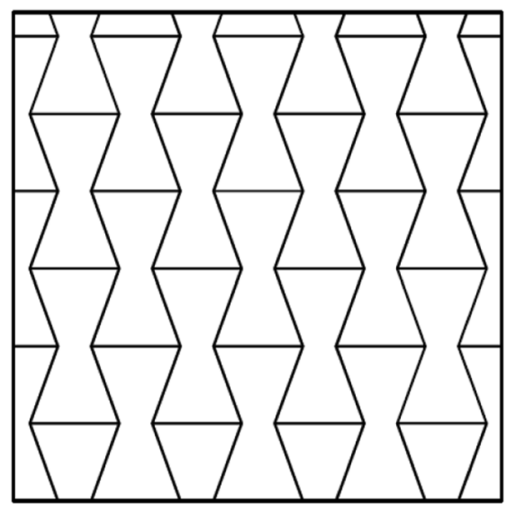

(d)

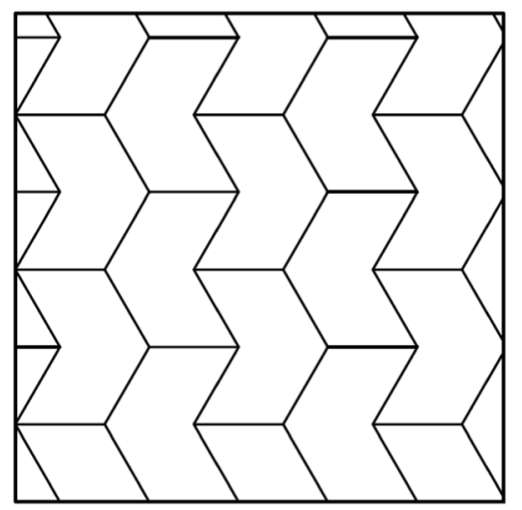

(g)

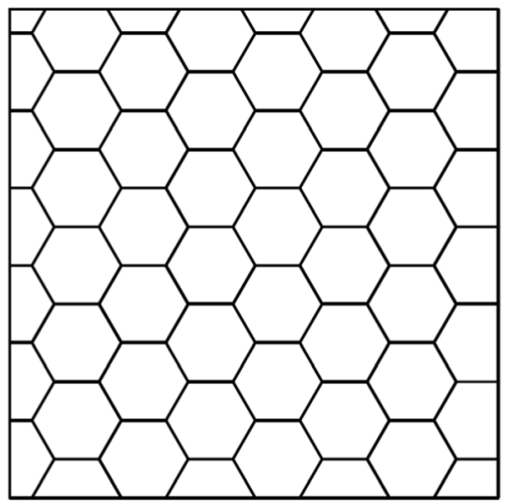

(b)

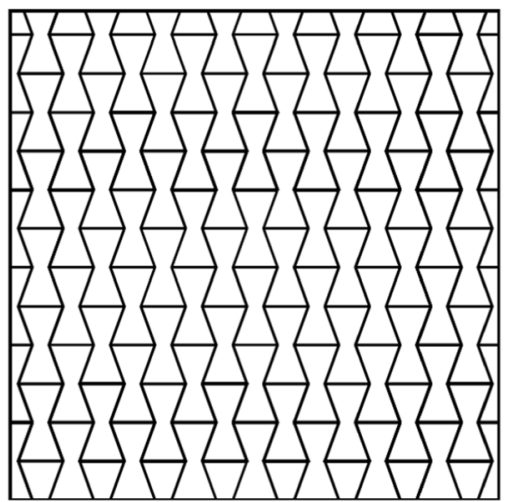

(e)

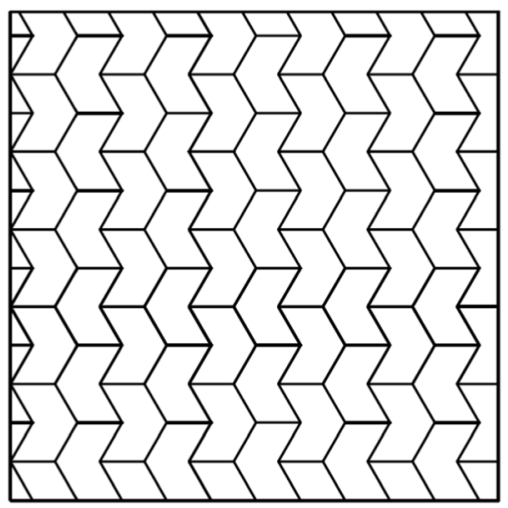

(h)

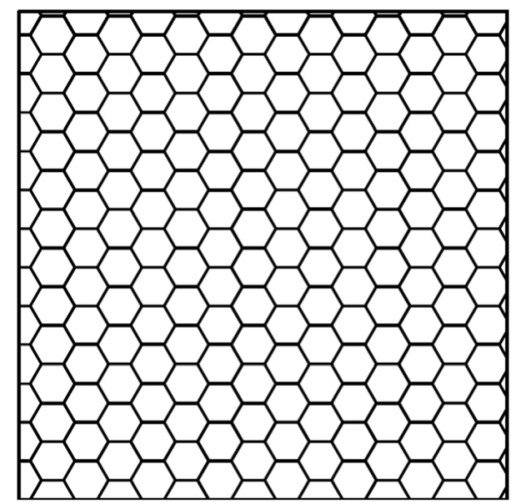

(c)



(f)

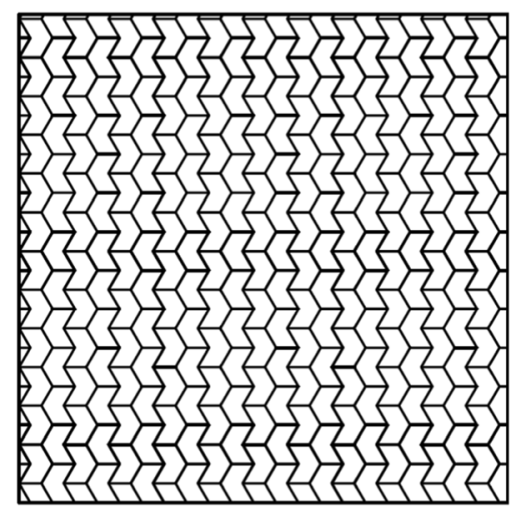

(i)

Fig. 2 Miscrostructure textures at different scales $s=1,0.5,0.25$ : a-c Regular Hexagon d-f Hourglass g-i Skew

scale-dependent, the definition of the RVE requires particular attention.

For the following analyses it is convenient to represent the constitutive matrix (8) in the form:

$\underline{C}=\left[\begin{array}{ll}\underline{A} & \underline{B} \\ \underline{B}^{T} & \underline{D}\end{array}\right]$
It is possible to show that only the constitutive components of $\underline{B}$ and $\underline{D}$ contain internal length scales, and this also justify the classification as 'implicitly' non local material [29].

In order to define the RVE, here by exploiting the results of numerical simulations performed comparing micropolar and discrete solutions (Sect. 3.1.2), with 
the latter solution assumed as benchmark, two different unit cells are considered.

\subsubsection{7-Blocks unit cell}

In the first case, the unit cell is constituted of one central block and six joints, therefore seven elements are considered (Fig. 3). The common edges interact by the medium point of the joint and the calculations of material constants are referred to the gravity center of the cell. is:

The constitutive matrix for regular hexagonal shape

$$
\begin{aligned}
\underline{A}_{r e g} & =\left[\begin{array}{cccc}
1189.7 & 170.0 & 0 & 0 \\
170.0 & 1189.7 & 0 & 0 \\
0 & 0 & 849.8 & 170.0 \\
0 & 0 & 170.0 & 849.8
\end{array}\right] ; \underline{B}_{r e g}=[\underline{0}] ; \\
\underline{D}_{\text {reg }}^{(s=1)} & =\left[\begin{array}{cc}
39.8 & 0 \\
0 & 28.5
\end{array}\right] ; \underline{D}_{\text {reg }}^{(s=0.5)}=\left[\begin{array}{cc}
10.0 & 0 \\
0 & 7.1
\end{array}\right] ; \underline{\sim}_{\text {reg }}^{(s=0.25)}=\left[\begin{array}{cc}
2.5 & 0 \\
0 & 1.8
\end{array}\right]
\end{aligned}
$$

It is worth noting that for the regular hexagons the constitutive matrix coefficients are such that $A_{1111}=A_{2222}, A_{1212}=A_{2121}$; there is no coupling between normal stresses and shear strains (tangential strains and longitudinal strains), microcouples $D_{12}=$ 0 as well as $\underline{B}_{r e g}=\underline{0}$. This configuration, invariant with respect to $\frac{\pi}{4}$ rotations, belongs to the orthotetragonal material symmetry class [10]. A small Poisson effect is shown. Furthermore, it is possible to observe that the components of $\underline{A}$ do not change with the decrease of scale, while the terms $\underline{B}$ and $\underline{D}$ depend on $s$; in particular, $\underline{D}_{\text {reg }}^{s=1} \approx 4 \underline{D}_{\text {reg }}^{s=0.5} \approx 16 \underline{D}_{\text {reg }}^{s=0.25}$. approximately,

The constitutive matrix for hourglass shape is:

$$
\begin{aligned}
\underline{A}_{\text {hour }} & =\left[\begin{array}{cccc}
584.4 & -126.1 & 0 & 0 \\
-126.1 & 2539.9 & 0 & 0 \\
0 & 0 & 1927.4 & -126.1 \\
0 & 0 & -126.1 & 346.7
\end{array}\right] ; \underline{B}_{\text {hour }}=[\underline{0}] ; \\
\underline{D}_{\text {hour }}^{(s=1)} & =\left[\begin{array}{cc}
6.6 & 0 \\
0 & 59.8
\end{array}\right] ; \underline{D}_{\text {hour }}^{(s=0.5)}=\left[\begin{array}{cc}
4.2 & 0 \\
0 & 15.0
\end{array}\right] ; \underline{D}_{\text {hour }}^{(s=0.25)}=\left[\begin{array}{cc}
1.0 & 0 \\
0 & 3.7
\end{array}\right]
\end{aligned}
$$

For the hourglass shape, the scale effect is always shown by the terms $\underline{D}$; in particular $\underline{D}_{\text {hour }}^{s=1} \approx 4 \underline{D}_{\text {hour }}^{s=0.5} \approx 16 \underline{D}_{\text {hour }}^{s=0.25}$. The negative terms $A_{1122}=A_{2211}$ show the auxetic behavior of the microstructure.

Finally, the constitutive matrix for skew shape is:

$$
\begin{aligned}
\underline{A}_{\text {skew }} & =\left[\begin{array}{cccc}
793.1 & 0 & 0 & 0 \\
0 & 1784.6 & 0 & 0 \\
0 & 0 & 1274.7 & 0 \\
0 & 0 & 0 & 566.5
\end{array}\right], \\
\underline{B}_{\text {skew }}^{T(s=1)} & =\left[\begin{array}{cccc}
0 & 0 & 0 & 0 \\
0 & 124.4 & 0 & 0
\end{array}\right] ; \\
\underline{B}_{\text {skew }}^{T(s=0.5)} & =\left[\begin{array}{cccc}
0 & 0 & 0 & 0 \\
0 & 62.2 & 0 & 0
\end{array}\right] ; \\
\underline{B}_{\text {skew }}^{T(s=0.25)} & =\left[\begin{array}{cccc}
0 & 0 & 0 & 0 \\
0 & 31.1 & 0 & 0
\end{array}\right] \\
\underline{D}_{\text {skew }}^{(s=1)} & =\left[\begin{array}{ccc}
26.6 & 0 \\
0 & 42.7
\end{array}\right] ; \underline{D}_{\text {skew }}^{(s=0.5)}=\left[\begin{array}{cc}
6.6 & 0 \\
0 & 10.7
\end{array}\right] ; \\
\underline{D}_{\text {skew }}^{(s=0.25)} & =\left[\begin{array}{cc}
1.7 & 0 \\
0 & 2.7
\end{array}\right]
\end{aligned}
$$

In this case there is no Poisson effect, $A_{1122}=A_{2211}=0$, and differently from the previous cases there is a coupling between elastic coefficients relating stresses and curvatures $(\underline{B} \neq \underline{0})$, in particular there is coupling between normal stress $\sigma_{22}$ and

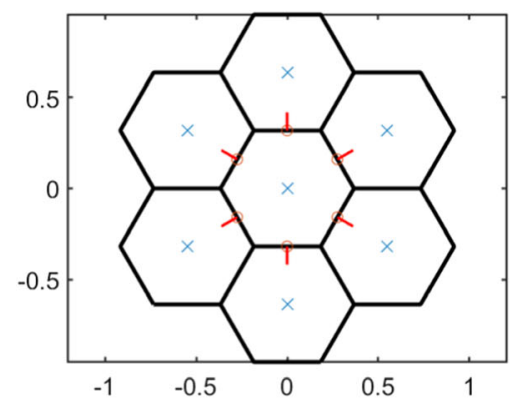

(a)



(b)

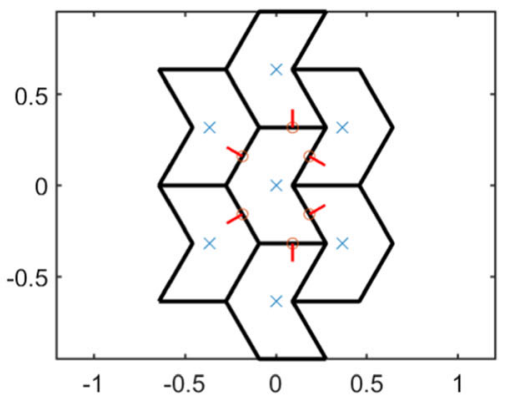

(c)

Fig. 3 Seven blocks unit cells: a Regular Hexagon b Hourglass c Skew 


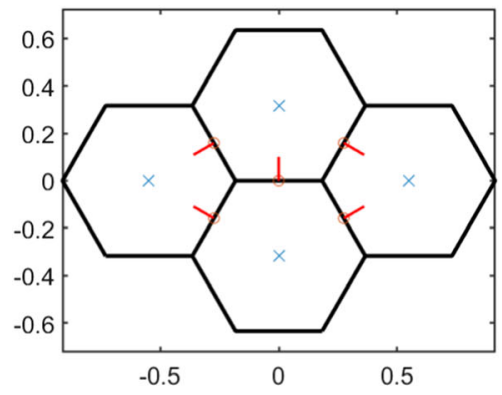

(a)

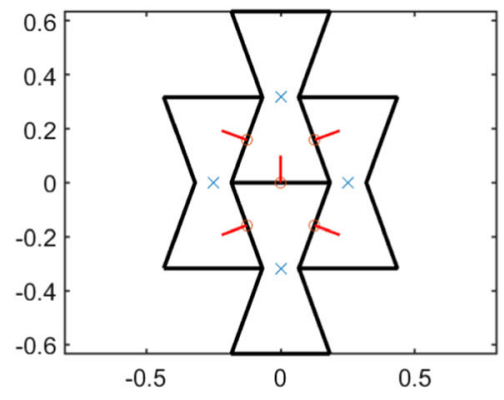

(b)

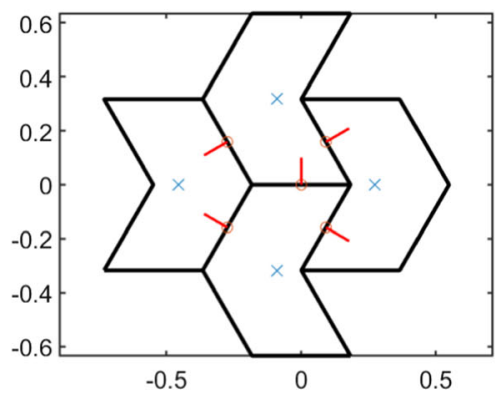

(c)

Fig. 4 Four blocks unit cells: a Regular Hexagon b Hourglass c Skew

curvature $k_{2}$. For this microstructure matrices $\underline{B}$ and $\underline{D}$ shows scale dependence, and in particular approximately $\underline{B}_{\text {skew }}^{s=1} \approx 2 \underline{B}_{\text {skew }}^{s=0.5} \approx 4 \underline{B}_{\text {skew }}^{s=0.25}, \underline{D}_{\text {skew }}^{s=1} \approx$ $4 \underline{D}_{\text {skew }}^{s=0.5} \approx 16 \underline{D}_{\text {skew }}^{s=0.25}$.

\subsubsection{4-Blocks unit cell}

In this case the unit cell considered is constituted of a system of four blocks with five joints, centered at the central joint (Fig. 4).

The constitutive matrices are different from the cases above. The new matrices are:

For the regular hexagons:



$$
\begin{aligned}
& \underline{D}_{r e g}^{(s=1)}=\left[\begin{array}{cc}
39.8 & 0 \\
0 & 20.9
\end{array}\right] ; \quad \underline{D}_{r e g}^{(s=0.5)}=\left[\begin{array}{cc}
10.0 & 0 \\
0 & 5.2
\end{array}\right] \text {; } \\
& \underline{D}_{r e g}^{(s=0.25)}=\left[\begin{array}{cc}
2.5 & 0 \\
0 & 1.3
\end{array}\right]
\end{aligned}
$$

It can be useful to observe that the constitutive matrices for the regular hexagon shapes are not orthotetragonal, the terms $A_{1111} \neq A_{2222}, A_{1212} \neq$ $A_{2121}$ as in the 7-blocks regular hexagon case because the joints are not in a symmetric configuration, invariant with respect to $\frac{\pi}{4}$, therefore in this case the symmetry is lost homogenizing from the discrete system to the continuous system in an equivalent energetic approach [10]. The micropolar term $\underline{D}$ is scale dependent with the same ratio as in the above case, approximately $\underline{D}_{\text {reg }}^{s=1} \approx 4 \underline{D}_{\text {reg }}^{s=0.5} \approx 16 \underline{D}_{\text {reg }}^{s=0.25}$.

The constitutive matrix for the hourglass shape is:

$$
\underline{A}_{\text {hour }}^{(s=1)}=\left[\begin{array}{cccc}
584.4 & -126.1 & 0 & 0 \\
-126.1 & 1547.2 & 0 & 0 \\
0 & 0 & 1431.1 & -126.1 \\
0 & 0 & -126.1 & 346.7
\end{array}\right]
$$

$$
\begin{aligned}
& \underline{B}_{\text {hour }}=[\underline{0}] ; \\
\underline{D}_{\text {hour }}^{(s=1)}= & {\left[\begin{array}{cc}
16.6 & 0 \\
0 & 43.2
\end{array}\right] ; \underline{D}_{\text {hour }}^{(s=0.5)}=\left[\begin{array}{cc}
4.2 & 0 \\
0 & 10.8
\end{array}\right] ; } \\
& \underline{D}_{\text {hour }}^{(s=0.25)}=\left[\begin{array}{cc}
1.0 & 0 \\
0 & 2.7
\end{array}\right]
\end{aligned}
$$

For the hourglass shape the matrices form are the same of the first case: in particular $\underline{D}_{\text {hour }}^{s=1} \approx 4 \underline{D}_{\text {hour }}^{s=0.5} \approx 16 \underline{D}_{\text {hour }}^{s=0.25}$. The negative terms $A_{1122}=A_{2211}$ show the auxetic behavior of the structure.

The constitutive matrix for the skew shape finally is: 


$$
\begin{aligned}
& \underline{A}_{\text {skew }}=\left[\begin{array}{cccc}
793.1 & 0 & 0 & 0 \\
0 & 1104.7 & 0 & 0 \\
0 & 0 & 934.8 & 0 \\
0 & 0 & 0 & 566.5
\end{array}\right] \text {; } \\
& \underline{B}_{\text {skew }}^{T(s=1)}=\left[\begin{array}{cccc}
0 & 0 & -85.5 & 51.8 \\
0 & 62.2 & 0 & 0
\end{array}\right] \text {; } \\
& \underline{B}_{\text {skew }}^{T(s=0.5)}=\left[\begin{array}{cccc}
0 & 0 & -42.8 & 25.9 \\
0 & 31.1 & 0 & 0
\end{array}\right] \text {; } \\
& \underline{B}_{\text {skew }}^{T(s=0.25)}=\left[\begin{array}{cccc}
0 & 0 & -21.4 & 13.0 \\
0 & 15.6 & 0 & 0
\end{array}\right] \\
& \underline{D}_{\text {skew }}^{(s=1)}=\left[\begin{array}{cc}
26.6 & 0 \\
0 & 31.3
\end{array}\right] ; \underline{D}_{\text {skew }}^{(s=0.5)}=\left[\begin{array}{cc}
6.6 & 0 \\
0 & 7.8
\end{array}\right] ; \\
& \underline{D}_{\text {skew }}^{(s=0.25)}=\left[\begin{array}{cc}
1.7 & 0 \\
0 & 2.0
\end{array}\right]
\end{aligned}
$$

For the skew shape the terms $B_{222}, B_{121}$ and $B_{211}$ are non null and, as in the skew 7-blocks case, a coupling between stresses/curvatures (microcouples/strains) is present. One again approximately: $\underline{B}_{\text {skew }}^{s=1} \approx 2 \underline{B}_{\text {skew }}^{s=0.5} \approx 4 \underline{B}_{\text {skew }}^{s=0.25}$, $\underline{D}_{\text {skew }}^{s=1} \approx 4 \underline{D}_{\text {skew }}^{s=0.5} \approx 16 \underline{D}_{\text {skew }}^{s=0.25}$.

Starting from the above constitutive matrices, using the same identification procedure the matrices for the classical Cauchy $2 \mathrm{D}$ problem have been calculated as follows:

$$
C=\left[\begin{array}{ccc}
A_{1111} & A_{1122} & 0 \\
A_{2211} & A_{2222} & 0 \\
0 & 0 & \frac{1}{2}\left[A_{1212}+A_{2121}\right]+A_{1221}
\end{array}\right]
$$

It is useful to note that the Cauchy constitutive matrix does not change with the scale variation, differently from the Cosserat matrix.

The question of the RVE definition is still open and the answer can depend on the type of microstructure and on the consideration that in an energy equivalent discrete-continuum transition the material symmetry class of the discrete assembly must be preserved [10].
Here a numerical criterion is adopted. In order to select the RVE for the homogenization procedure here adopted, the external load work of the static analysis case studied in Sect. 5 has been calculated for the discrete assembly and for the micropolar model of both 7-blocks and 4-blocks unit cells. The comparison refers to the static analysis of a rectangular panel where the microstructure factor scale is 0.5 and the results are reported in Table 1 . The analyses relative to the the scale 1 and scale 0.25 are omitted because they give analogously consequences. Basing on these results, the 7-blocks unit cell of Subsect. 3.1.1 has been selected as the RVE for the numerical simulations performed; furthermore the Cauchy constitutive matrices are calculated from the matrices showed in Subsect. 3.1.1.

\section{Numerical implementation}

In the next subsections a numerical implementation of the micropolar theory is resumed and the discrete models adopted for the simulations are described.

\subsection{Continuum model}

The present finite element framework is based on the previous works [15, 38]. The finite element enforces an approximation through nodal kinematic parameters as:

$\underline{u}=\underline{\mathcal{N}} \underline{d}^{e}$

The displacement vector for the present problem is given by:

$\underline{d}^{e T}=\left[\begin{array}{lllllllll}u_{1}^{1} & \ldots & u_{1}^{4} & u_{2}^{1} & \ldots & u_{2}^{4} & \omega^{1} & \ldots & \omega^{4}\end{array}\right]$

\begin{tabular}{|c|c|c|c|c|c|}
\hline \\
\hline Shape & Discrete & \multicolumn{4}{|c|}{$\frac{\text { Scale } 0.5 \text { - External work }}{\text { Shane }}$} \\
\hline Regular & 0.0796 & 0.0722 & -9.35 & 0.113 & 41.7 \\
\hline Hourglass & 0.0569 & 0.0484 & -14.9 & 0.0756 & 33.0 \\
\hline Skew & 0.0650 & 0.0556 & -14.5 & 0.0860 & 32.3 \\
\hline
\end{tabular}

the finite element has 12 degrees of freedom. The matrix of the shape functions takes the form $[37,38]$ :
Table 1 External work results for the static analyses (Sect. 5) 


$$
\underline{\mathcal{N}}=\left[\begin{array}{ccc}
\underline{N} & \underline{0} & \underline{0} \\
\underline{0} & \underline{N} & \underline{0} \\
\underline{0} & \underline{0} & \underline{N}
\end{array}\right]
$$

where $\underline{N}$ is the vector of the linear Lagrangian shape functions. The internal work becomes [39]:

$$
\begin{aligned}
\delta U & =\delta \underline{d}^{e T} h \int_{A}(\underline{D} \underline{\mathcal{N}})^{T} \underline{C}(\underline{D} \underline{\mathcal{N}}) d A \underline{d}^{e} \\
& =\delta \underline{d}^{e T} h \int_{A} \underline{B}^{T} \underline{C} \underline{B} d A \underline{d}^{e}
\end{aligned}
$$

where $\underline{B}=\underline{D} \underline{\mathcal{N}}$, thus the element stiffness matrix is:

$$
\underline{K}^{e}=\int_{A} \underline{B}^{T} \underline{C} \underline{B} d A
$$

The potential of loads becomes:

$$
\begin{aligned}
\delta V= & -\delta \underline{d}^{e T} h \int_{A} \underline{N}^{T} \underline{f} d V-\delta \underline{\delta}^{e T} \\
& \int_{S} \underline{N}^{T} \underline{p} d S=-\delta \underline{d}^{e T}\left(\underline{F}^{e}+\underline{P}^{e}\right)
\end{aligned}
$$

where $\underline{F}^{e}$ and $\underline{P}^{e}$ are volume and surface force vectors respectively. Linear shape functions are considered for the present implementation with reduced integration for the micro-rotation $\omega$ [40]. To perform reduced integration the strain vector has to be reordered by separating strain terms which are fully integrated and the ones for which reduced integration is applied.

Once the problem is solved in terms of displacements other quantities such as stresses and relative rotation have to be post computed [40].

\subsection{Discrete model}

The discrete solution has been obtained using the FEM software ABAQUS; because of the complexity to build the model through the program interface, Python script codes have been prepared. Static analyses with three different discrete models are presented as a benchmark for the numerical study in order to show the goodness of the adopted homogenization technique [10].

\subsubsection{Spring model}

In the first model three linear elastic springs, longitudinal, transversal and rotational, are assigned at the center of each joints. For the edges parallel to axis $x$ or $y$, springs are represented in the joint local reference system. The scripts are structured in the following principal steps:

1. The geometry of the hexagonal shape is defined;

2. The material properties are defined;

3. The parts of the model are created;

4. The boundary blocks of the panel are cut to define the rectangular geometry panel;

5. Edges are partitioned to define the middle point for each joint;

6. Rotational and translational springs are added for all internal joints.

The single block must behave as a rigid body, therefore the material is assigned as an elastic isotropic behavior with a high value of the Young modulus with respect to the joint stiffness in particular $E=100$ MPa. The normal stiffness is represented by the $k_{11}$ term in reference to the normal direction to the contact area and the tangential stiffness by the term $k_{22}$ along the contact area plane in the local system (Fig. 5).

Energetic equivalence is used to carry out the rotational stiffness [39]:

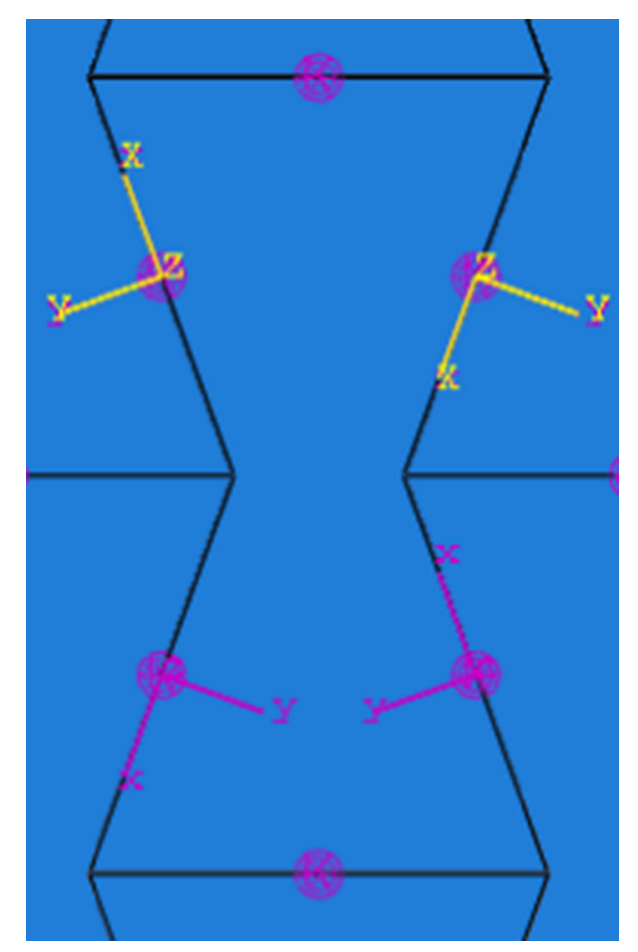

Fig. 5 Example of a single tile for the hourglass shape with elastic springs and local reference systems 
$k_{r}=k_{11} \frac{d^{2}}{4}$

where $d$ is the generic edge length of the single tile. Finally the boundary conditions, loads and the mesh of model are manually input.

\subsubsection{Contact interaction}

The elements, the joints and the texture of the discrete model are realized through Python script. However, in the present implementation the interactions between rigid blocks is considered as distributed contact with the ABAQUS interaction option "surface interaction". The pressure-overclosure relationship are prescribed by using a linear elastic law: the surfaces transmit contact pressure when the overclosure between them, measured in the contact (normal) direction, is greater than zero. The linear pressureoverclosure relationship is identical to a tabular relationship with two data points, where the first point is located at the origin, it is necessary to specify the slope of the pressure-overclosure relationship, $k$. For the the tangential direction it is set the friction formulation as "frictionless" to consider the case with no tangential stiffness, in this way ABAQUS assumes that surfaces in contact slide freely without friction. It is considered this particular case for the difficulty to define a linear elastic tangential behavior as done for the normal direction. Various numerical tests have been done and the results are very close to the springs model, obviously with only a normal and rotational springs; the quality of the test indicates the right calculation for the rotational stiffness.

\subsubsection{Elastic Interfaces}

The third approach considers linear elastic interfaces among the blocks. Two parts in ABAQUS are created: one for the rigid blocks and one for the elastic interfaces. The mechanical properties of the materials were defined for the rigid block as orthotropic behavior with a high Young modulus and for the elastic interfaces engineering constants are set in order to fictitiously model a frictionless elastic interface with $G_{13}=G_{23}=G_{12}=0$. The Young modulus was calculated as $E A / l=k_{n} s / l$, therefore $E=k_{n} s / A$ where: $E$ is the Young modulus, $A$ is the interface contact area, $A=t d$; with $d$ the edge length (because in this case the contact is developed along the whole surfaces) and $t$ is the panel thickness. $k_{n}$ is the normal spring stiffness and $s$ thickness of the layer. Thus, assuming a unitary thickness $t=1$, the modulus becomes:

$E=\frac{k_{n} s}{d}$

The Young modulus $E$ increases with the decrease of the scale. Subsequently from the blocks and interfaces parts two instances were created: then they were bonded by the "tie constraints", that give them the same displacements at the contact interface. For explanatory purposes, using the three discrete systems previously described, in Fig. 6 a comparison of the vertical displacements for a panel clamped at the bottom with a vertical concentrated force at the center of the upper edge is reported.

From the vertical displacement contour plot a greater similarly between the spring and contact models can be detected, in particular for the panel region far from the applied load. The discrete model with elastic springs has been used for a comparison with the equivalent micropolar continuum model: this case is closer to the theoretical discrete model presented in [13]. Moreover, the springs model is faster with respect to the model with contact interactions for which the computational cost increases exponentially with the scale reduction. In addition, contact interfaces leads to a non linear model on the contrary springs interfaces are linear.

\section{Simulations}

In this section, the behavior of $2 \mathrm{D}$ rectangular panels with different microstructural shape and different scales is investigated in Fig. 7, the panel is clamped at the base and a vertical load is applied at the top side center, for a footprint length equals $a=L_{x} / 4, L_{x}$ being the base length. The resultant of the load is a force of intensity $P=10 \mathrm{~N}$, the stiffness terms are $k_{11}=785$ $\mathrm{N} / \mathrm{m}$ and $k_{22}=392.5 \mathrm{~N} / \mathrm{m}$ and the length of panel sides are $L_{x}=6.6 \mathrm{~m}, L_{y}=7.7 \mathrm{~m}$ for the regular 

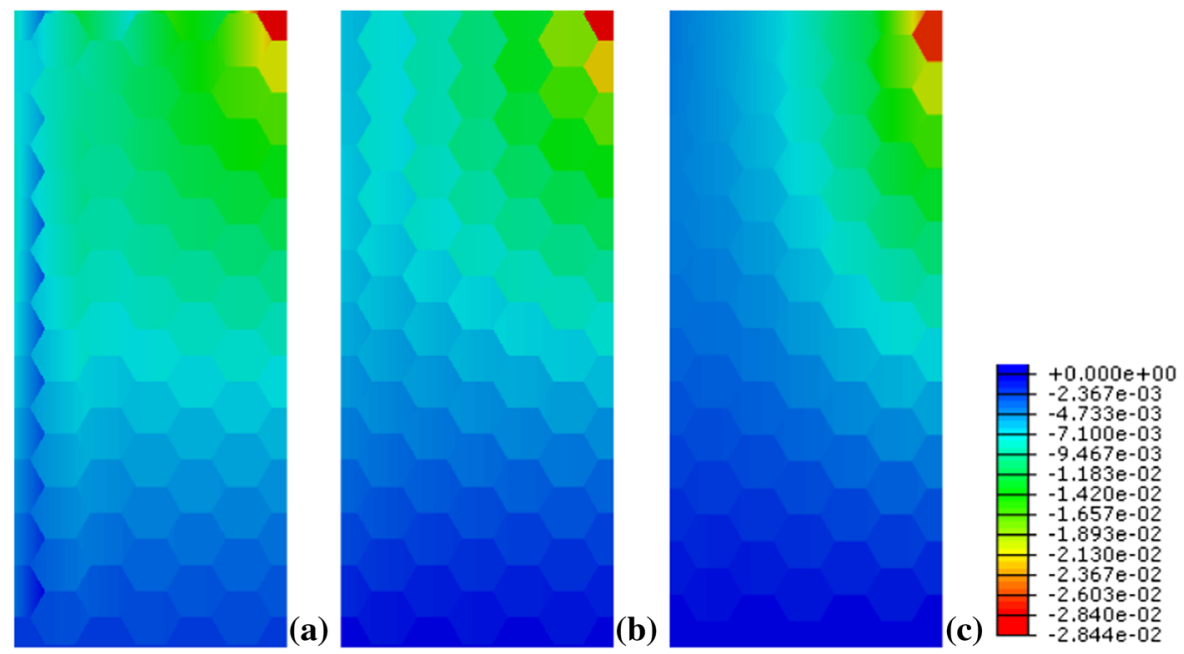

Fig. 6 Vertical displacement component for discrete models with regular hexagonal shape scale $s=1$ and vertical load applied at the top. a Elastic springs b Elastic contact $\mathbf{c}$ Elastic interface

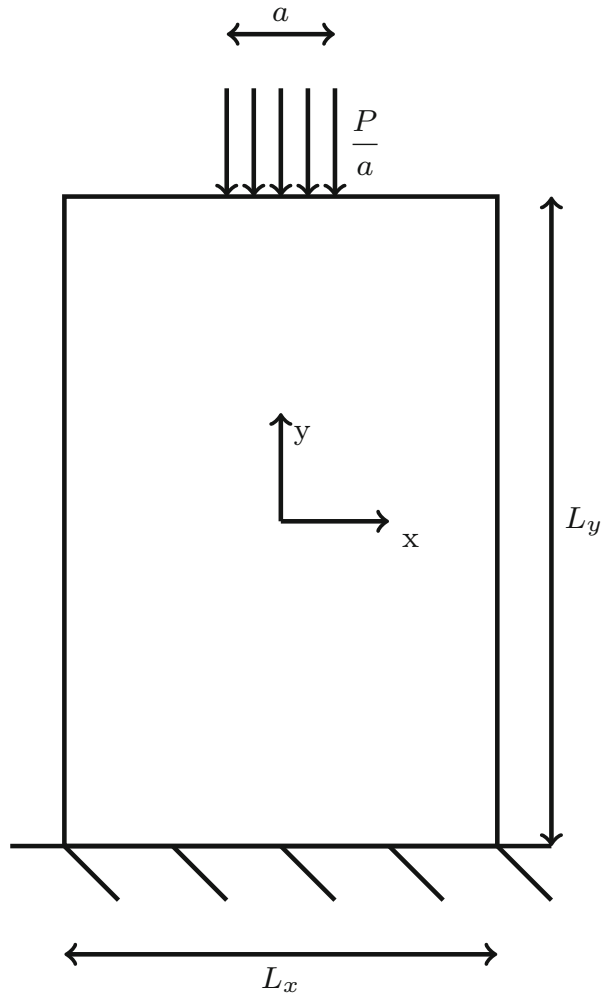

Fig. 7 Rectangular panel, static scheme

hexagonal shape, $L_{x}=5 \mathrm{~m}, L_{y}=7.7 \mathrm{~m}$ for the hourglass shape and $L_{x}=5.85 \mathrm{~m}, L_{y}=7.7 \mathrm{~m}$ for the skew shape.
In the next sections, a displacement field comparison among the discrete spring model and the micropolar and classical continuum models is reported.

\subsection{Regular hexagonal shape}

Horizontal components of displacement are shown (Fig. 8): the contour plot of the micropolar continuum is in line with the discrete system. There are not evident differences among the Cosserat and Cauchy model. This is expected because of the orthotetragonal configuration of regular hexagons, that implies corresponding results among all the models [14].

For vertical displacements the micropolar continuum contour plot is in line with the discrete system behavior, once again because of the orthotetragonal configuration no evident differences among the Cosserat and Cauchy model are revealed and their differences vanish when the microstructure scale factor become smaller (Fig. 9).

\subsection{Hourglass shape}

Horizontal displacements are shown (Fig. 10): the contour plot of the micropolar continua is in line with the discrete system. At the top of the panel a smaller maximum displacement increase can be noted with the scale reduction for the discrete system. 
Fig. 8 Horizontal

displacement component, regular hexagonal shape
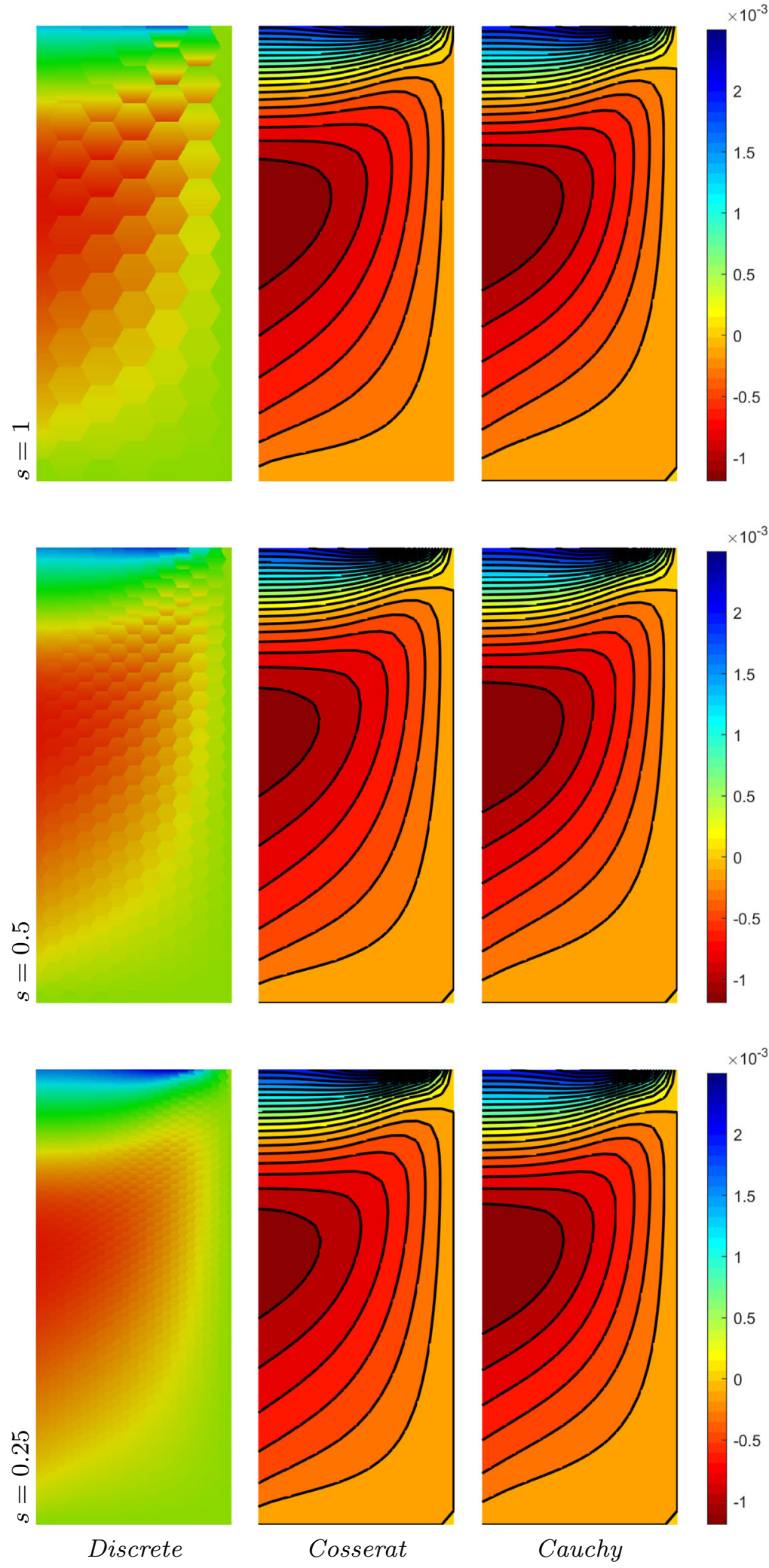
Fig. 9 Vertical

displacement component, regular hexagonal shape
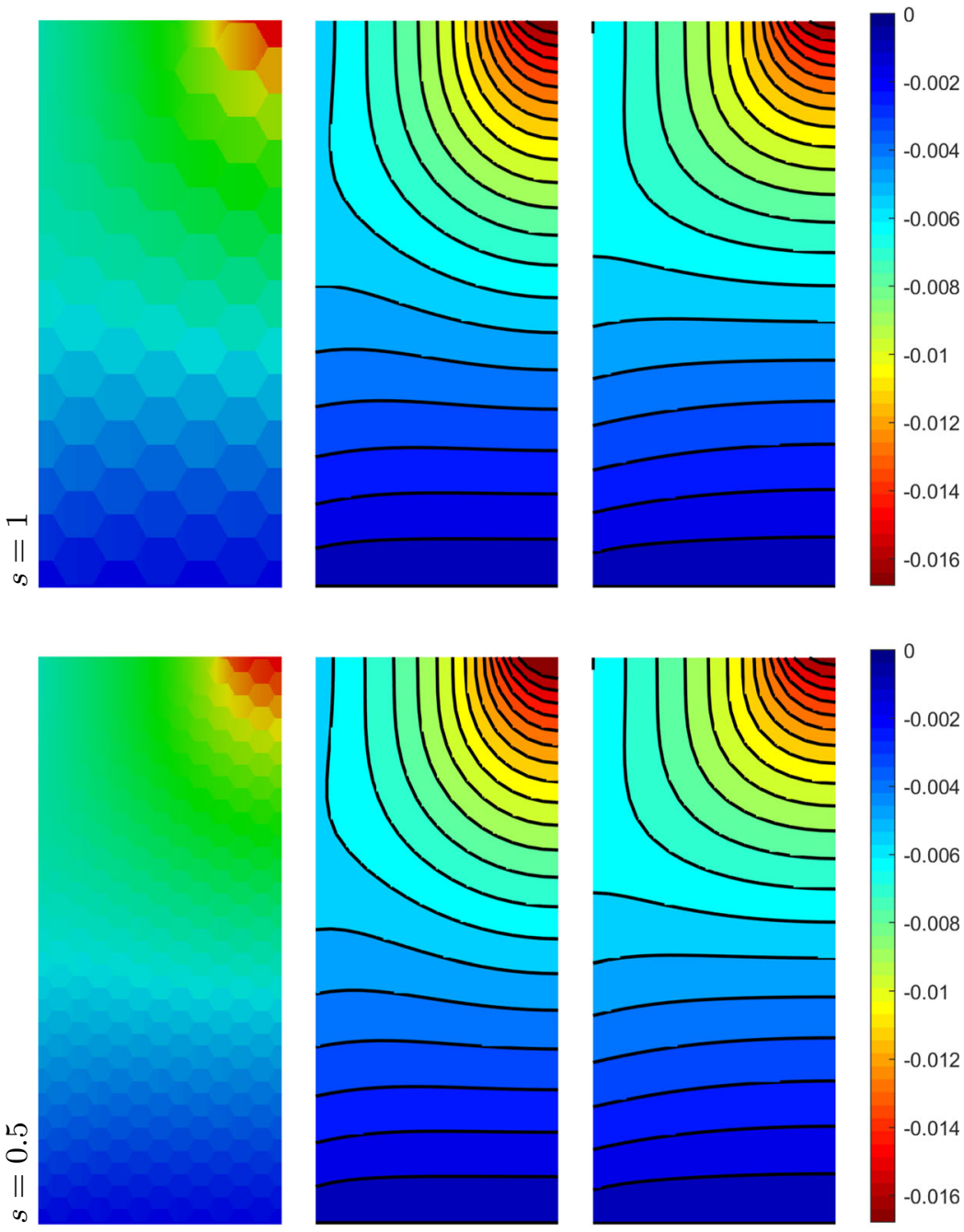

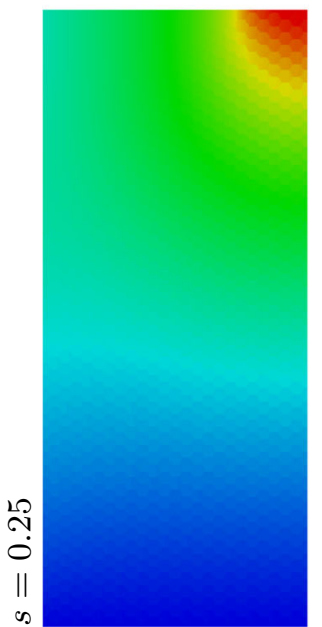

Discrete

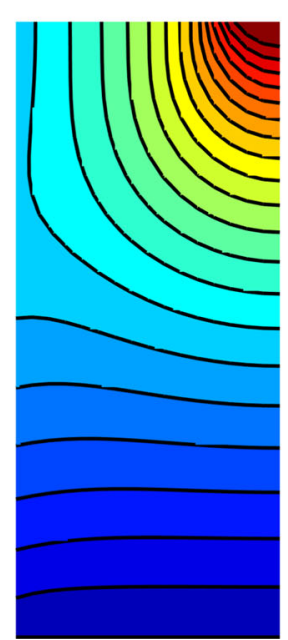

Cosserat

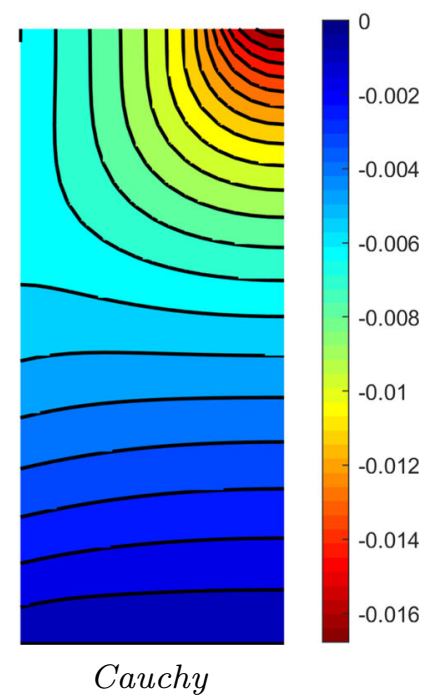

Cauchy 
Fig. 10 Horizontal

displacement component, hourglass shape
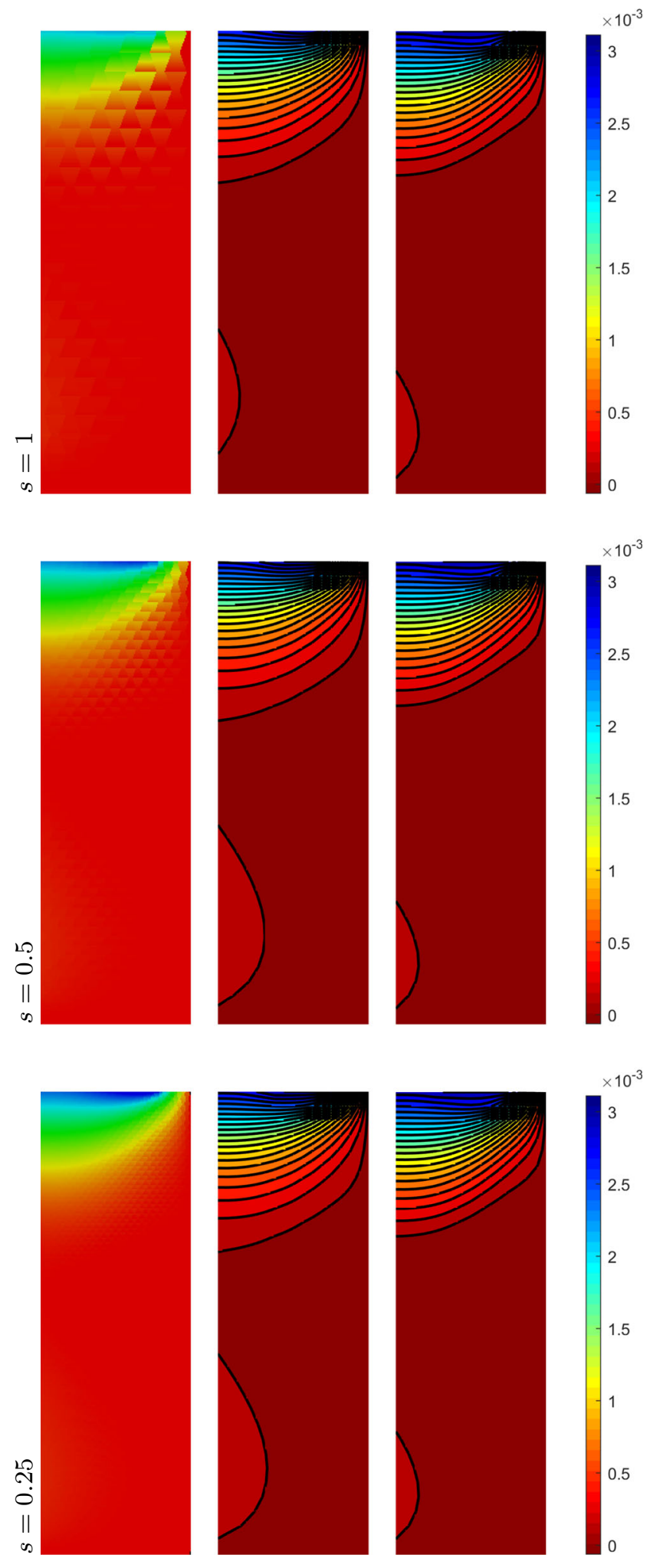

Cosserat

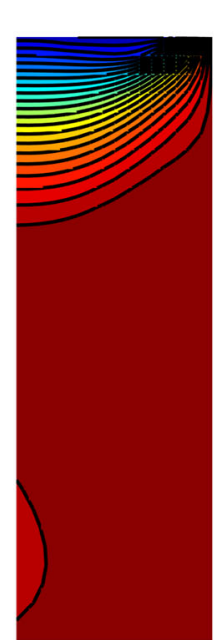

Cauchy

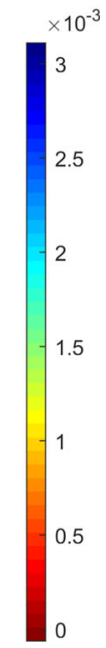


Fig. 11 Vertical

displacement component,

hourglass shape
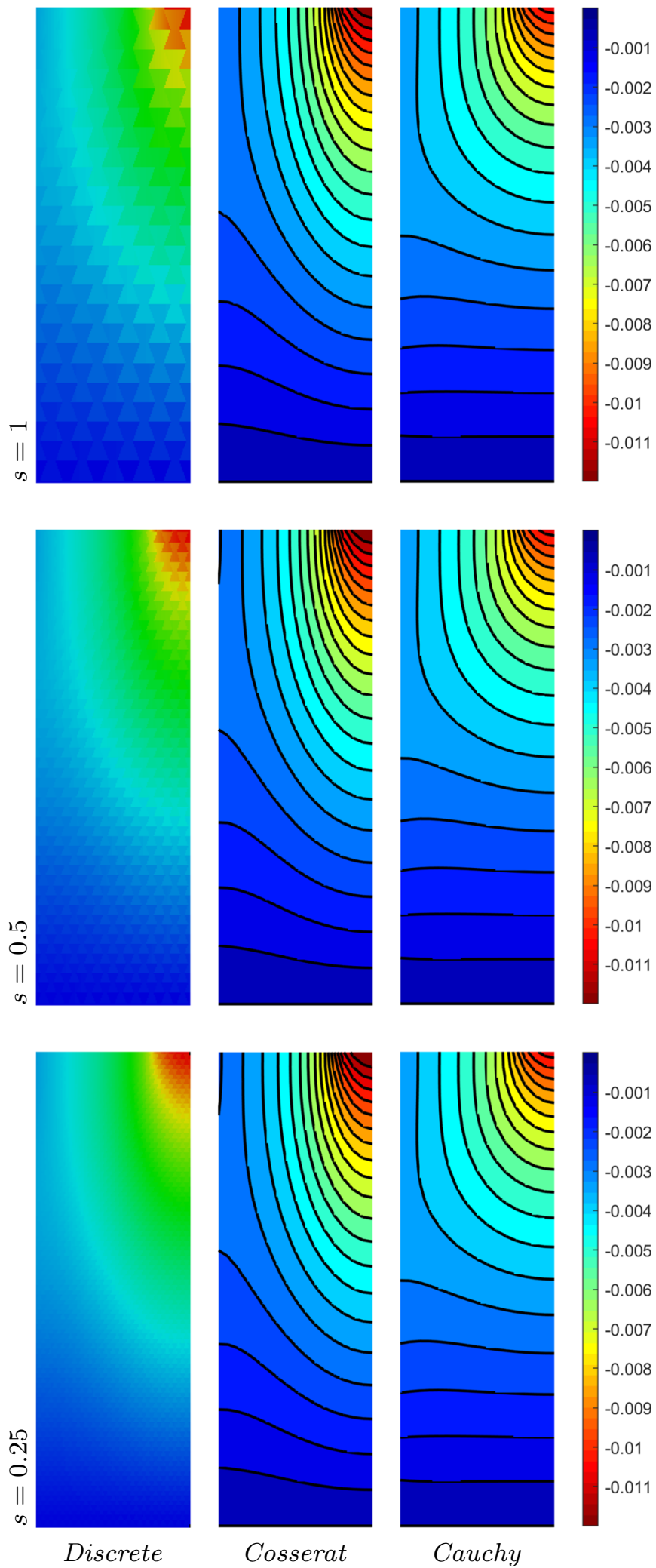

Cosserat
$-0.002$

$-0.003$

.004

.005

.008

01

$-0.011$

.002

.003

.004

0.005

.006

007

009

$-0.01$

$-0.011$

$-0.002$

$-0.003$

$-0.004$

$-0.005$

$-0.006$

0.007

.008

$-0.009$

$-0.01$

$-0.011$

Cauchy 
Fig. 12 Horizontal

displacement component, skew shape
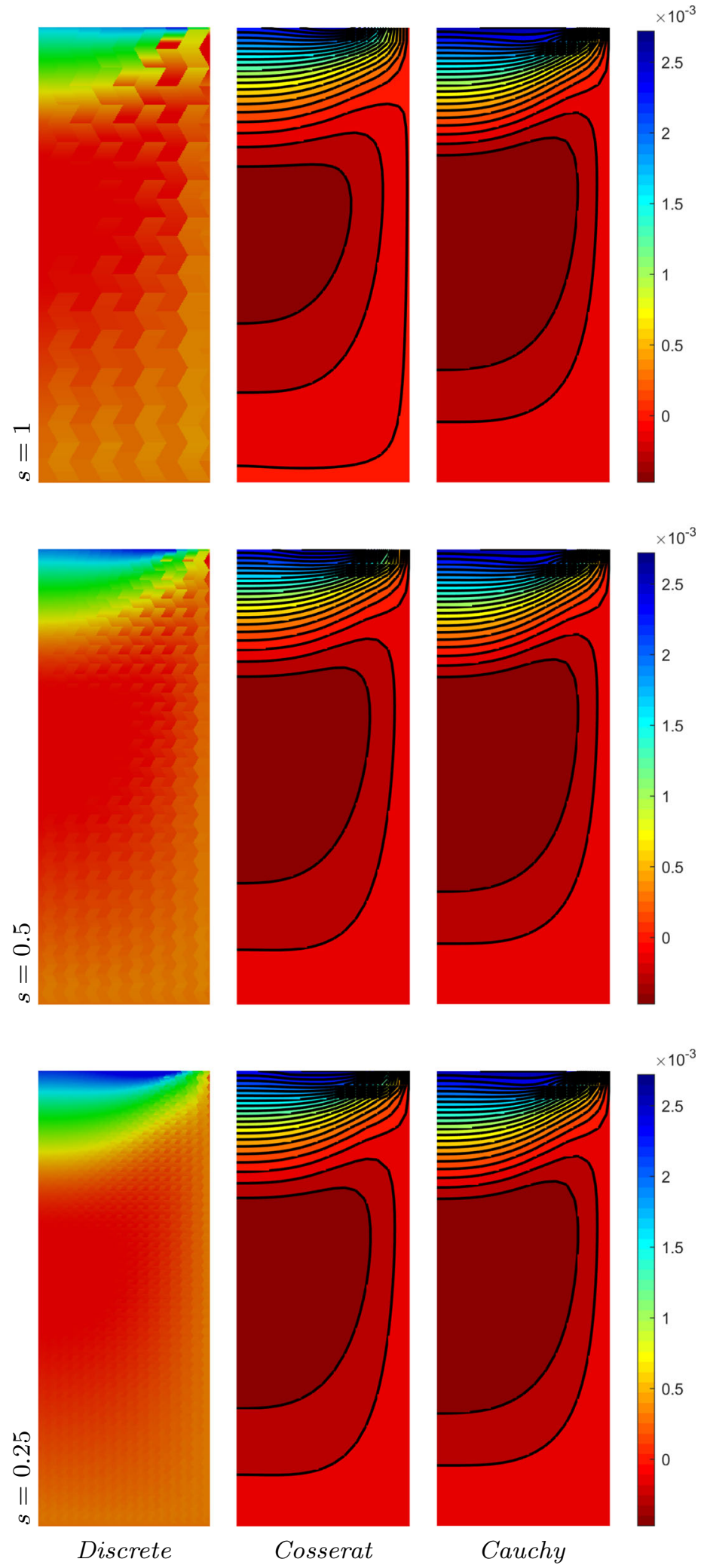

䌼 Springer 
Fig. 13 Vertical

displacement component, skew shape
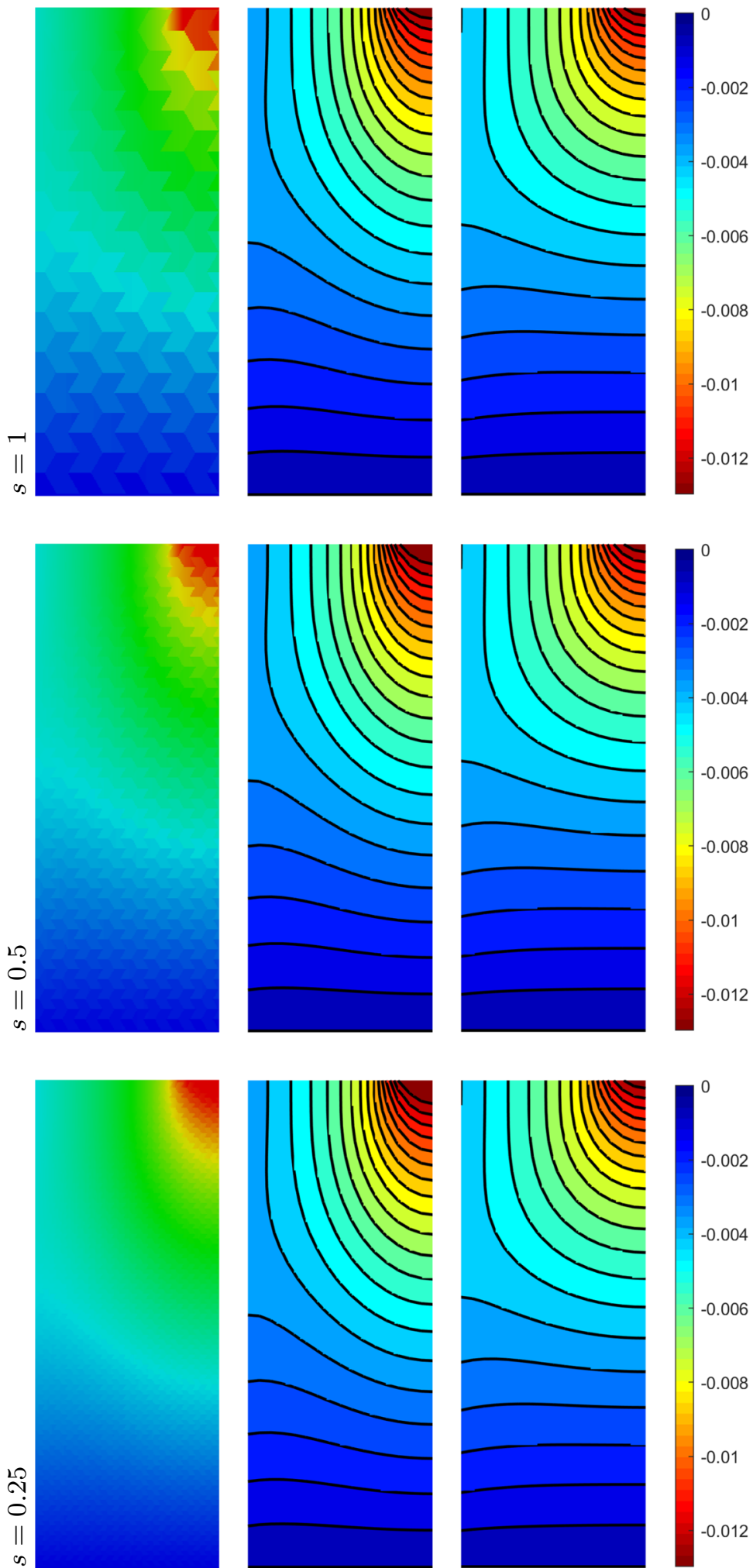

Discrete

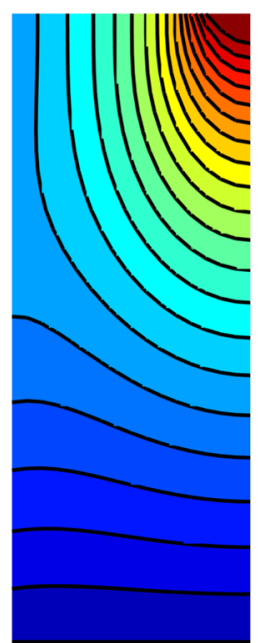

Cosserat

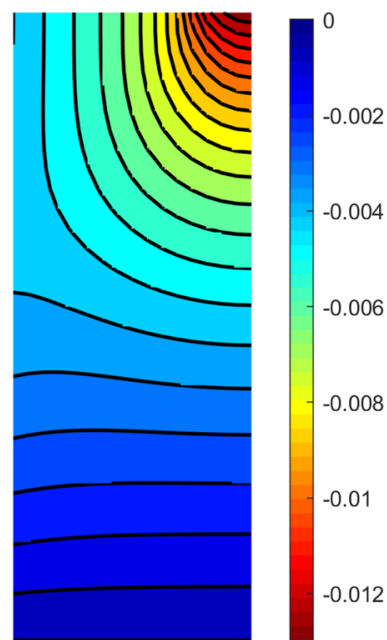

Cauchy 
Also for the vertical displacements the micropolar continuum contour plot is in line with the discrete system behavior (Fig. 11). The effect of negative Poisson ratio is visible in this configuration which displays concentrated vertical displacements which are close to zero far from the applied load, this is due to the horizontal contraction of the material. A major displacement distribution can be noted for the micropolar model respect to the classical model: moreover, for the hourglass shape a major percolation [58] is visible for the Cosserat model respect to the Cauchy continuum and this behavior is closer to the discrete system plot.

\subsection{Skew shape}

The micropolar continuum model reproduces accurately the discrete system behavior, once again for the horizontal displacements there are not obvious differences among the three continuum models. For the discrete system, with the scale reduction a smaller displacement magnitude increase can be noted at the panel top (Fig. 12).

Also for the vertical displacements field the micropolar continuum gives a faithful representation for the discrete system contour plot and once again the Cosserat model reveal a major distribution of the level curves in comparison with the Cauchy model (Fig. 13). Lastly as already shown for the hourglass shape, in the skew shape contour plot the percolation effect is more evident for the micropolar model.

\section{Conclusion}

The present work investigates the static behavior of composite materials with three types of hexagonal microstructures as equivalent micropolar media. The comparison among the discrete model and the continuum models highlighted that assemblies of regular hexagons have an orthotetragonal behavior, therefore there is only a weak dependence on the scale, as in the case of materials with elements of small size, and it has been shown that their homogenized behavior is close to the behavior of classical elastic bodies. For the hourglass and skew configuration, the scale dependence is more evident both for the discrete system and for the micropolar continuum, which properly accounts for the non-symmetries as well as size effects exhibited by the discrete system, as consequence, this last should be preferred in this cases. It is then highlighted the scale dependence of the discrete system and how the Cosserat model takes into account it, differently for the Cauchy model, and how to consider the asymmetric behavior of anisotropic materials is fundamental.

Moreover, the static analyses comparison shows that the homogenization technique can be generalized for hexagonal geometry microstructure, both for concave shapes and convex shapes [10, 13, 35]. For the study of particle composites the possibility to use a proven homogenization technique involves in a faster and efficient computational modeling. Furthermore three discrete models have been used to study composite materials described as materials made of rigid particles and elastic interfaces. The comparison between them show the right assumption to calculate the interface rotational stiffness. The elastic spring model is a linear model more faster for the numerical simulation compared to other two and it well represent the mathematical theoretical model adopted for the discrete system representation. Finally, other geometries can be investigated in the future comparing the discrete system and the continuum model and as well as the dynamic case can be studied.

Acknowledgements This research was supported by the Italian Ministry of University and Research, P.R.I.N. 2015, project 2015JW9NJT Advanced mechanical modeling of new materials and structures for the solution of 2020 Horizon challenges, Sapienza Research Unit (grant B86J16002300001) by Sapienza University, grant 2016 (B82F16005920005), and P.R.I.N. 2017 no. 20172017HFPKZY (cup: B86J16002300001).

Funding Open access funding provided by Università degli Studi di Roma La Sapienza within the CRUI-CARE Agreement. This study was funded by the Italian Ministry of University and Research, P.R.I.N. 2015 (grant number 2015JW9NJT). This study was funded by Sapienza Research Unit by Sapienza University (grant number B86J16002300001). This study was funded by Sapienza Research Unit by Sapienza University (grant number B82F16005920005). This study was funded by the Italian Ministry of University and Research, P.R.I.N. 2017 (grant number no. 2017HFPKZY, cup: B86J16002300001); Sapienza Research Grants "Progetti Grandi" 2018 (B81G19000060005).

\section{Declarations}

Conflict of interest The authors declare that they have no conflict of interest. 
Open Access This article is licensed under a Creative Commons Attribution 4.0 International License, which permits use, sharing, adaptation, distribution and reproduction in any medium or format, as long as you give appropriate credit to the original author(s) and the source, provide a link to the Creative Commons licence, and indicate if changes were made. The images or other third party material in this article are included in the article's Creative Commons licence, unless indicated otherwise in a credit line to the material. If material is not included in the article's Creative Commons licence and your intended use is not permitted by statutory regulation or exceeds the permitted use, you will need to obtain permission directly from the copyright holder. To view a copy of this licence, visit http://creativecommons.org/licenses/by/4.0/.

\section{References}

1. Lotfi HR, Shing PB (1994) Interface model applied to fracture of masonry structures. J Struct Eng 120(1):63-80

2. Lourenço PB, Rots JG (1997) Multisurface interface model for analysis of masonry structures. $J$ Eng Mech 123(7):660-668

3. Yang D, Sheng Y, Ye J, Tan Y (2010) Discrete element modeling of the microbond test of fiber reinforced composite. Comput Mater Sci 49(2):253-259

4. Baraldi D, Reccia E, Cecchi A (2018) In plane loaded masonry walls: Dem and fem/dem models. A critical review. Meccanica 53(7):1613-1628

5. Pepe M, Pingaro M, Trovalusci P, Reccia E, Leonetti L (2020) Micromodels for the in-plane failure analysis of masonry walls: limit analysis, FEM and FEM/DEM approaches. Frattura ed Integritá Strutturale 14(14):504-516

6. Nemat-Nasser S, Hori M (2013) Micromechanics: overall properties of heterogeneous materials. Elsevier, Amsterdam

7. Bigoni D, Drugan WJ (2007) Analytical derivation of Cosserat moduli via homogenization of heterogeneous elastic materials. J Appl Mech 74(4):741-753. https://doi. org/10.1115/1.2711225

8. Ehlers W (2011) Recovering micropolar continua from particle mechanics by use of homogenisation strategies. In: Mueller-Hoeppe D, Löhnert S, Reese S (eds) Recent developments and innovative applications in computational mechanics, Springer, pp 179-189. https://doi.org/10.1007/ 978-3-642-17484-1

9. Li X, Liu Q, Zhang J (2010) A micro-macro homogenization approach for discrete particle assembly-Cosserat continuum modeling of granular materials. Int J Solids Struct 47(2):291-303

10. Trovalusci P, Masiani R (1999) Material symmetries of micropolar continua equivalent to lattices. Int J Solids Struct 36(14):2091-2108

11. Trovalusci P (2014) Molecular approaches for multifield Continua: origins and current developments. In: Sadowski T, Trovalusci P (eds) Multiscale modeling of complex materials. CISM international centre for mechanical sciences, vol 556. Springer, Vienna. https://doi.org/10.1007/ 978-3-7091-1812-2_7
12. Trovalusci $P$ (2015) Materials with internal structure: multiscale and multifield modeling and simulation. Springer, Berlin

13. Masiani R, Trovalusci P (1996) Cosserat and Cauchy materials as continuum models of brick masonry. Meccanica 31(4):421-432

14. Trovalusci P, Pau A (2014) Derivation of microstructured continua from lattice systems via principle of virtual works: the case of masonry-like materials as micropolar, second gradient and classical continua. Acta Mechanica 225(1):157-177

15. Fantuzzi N, Trovalusci P, Dharasura S (2019) Mechanical behavior of anisotropic composite materials as micropolar continua. Front Mater 6:59

16. Kunin IA (1984) On foundations of the theory of elastic media with microstructure. Int J Eng Sci 22(8-10):969-978

17. I. A. Kunin (1968) The theory of elastic media with microstructure and the theory of dislocations. In: Altenbach $\mathrm{H}$, Maugin GA, Erofeev V (eds) Mechanics of generalized continua, Springer, pp. 321-329. https://doi.org/10.1007/ 978-3-642-19219-7

18. Sluys LJ, De Borst R, Mühlhaus HB (1993) Wave propagation, localization and dispersion in a gradient-dependent medium. Int J Solids Struct 30(9):1153-1171

19. Trovalusci P, Varano V, Rega G (2010) A generalized continuum formulation for composite microcracked materials and wave propagation in a bar. $\mathrm{J}$ Appld Mech 77(6):061002

20. Reda H, Rahali Y, Ganghoffer J, Lakiss H (2016) Wave propagation in $3 \mathrm{~d}$ viscoelastic auxetic and textile materials by homogenized continuum micropolar models. Compos Struct 141:328-345

21. Settimi V, Trovalusci P, Rega G (2019) Dynamical properties of a composite microcracked bar based on a generalized continuum formulation. Contin Mech Thermodyn 31(6):1627-1644

22. Eremeyev VA, Rosi G, Naili S (2020) Transverse surface waves on a cylindrical surface with coating. Int J Eng Sci 147:103188

23. Nowacki W (1972) Theory of micropolar elasticity. Springer, Berlin

24. Eringen AC (1999) Theory of micropolar elasticity. In: Microcontinuum field theories, Springer, New York, NY. https://doi.org/10.1007/978-1-4612-0555-5_5

25. Altenbach J, Altenbach H, Eremeyev VA (2010) On generalized Cosserat-type theories of plates and shells: a short review and bibliography. Archive Appl Mech 80(1):73-92

26. Altenbach H, Eremeyev VA (2012) Generalized continuafrom the theory to engineering applications, vol 541. Springer, Berlin

27. Capriz G (2013) Continua with microstructure, vol 35. Springer, Berlin

28. Tuna M, Kirca M, Trovalusci P (2019) Deformation of atomic models and their equivalent continuum counterparts using Eringen's two-phase local/nonlocal model. Mech Res Commun 97:26-32

29. Tuna M, Trovalusci P (2020) Scale dependent continuum approaches for discontinuous assemblies:'explicit'and 'implicit'non-local models. Mech Res Commun 103:103461 
30. Forest S, Sab K (1998) Cosserat overall modeling of heterogeneous materials. Mech Res Commun 25(4):449-454

31. Forest S, Dendievel R, Canova GR (1999) Estimating the overall properties of heterogeneous Cosserat materials. Model Simul Mater Sci Eng 7(5):829

32. Forest S, Barbe F, Cailletaud G (2000) Cosserat modelling of size effects in the mechanical behaviour of polycrystals and multi-phase materials. Int $J$ Solids Struct 37(46-47):7105-7126

33. Ostoja-Starzewski M, Boccara SD, Jasiuk I (1999) Couplestress moduli and characteristic length of a two-phase composite. Mech Res Commun 26(4):387-396

34. Trovalusci P, Masiani R (2005) A multifield model for blocky materials based on multiscale description. Int $\mathrm{J}$ Solids Struct 42(21-22):5778-5794

35. Trovalusci P, Masiani R (2003) Non-linear micropolar and classical continua for anisotropic discontinuous materials. Int J Solids Struct 40(5):1281-1297

36. Trovalusci P, Sansalone V (2007) A numerical investigation of structure-property relations in fiber composite materials. Int J Multiscale Comput Eng 5(2):141-152

37. Fantuzzi N, Leonetti L, Trovalusci P, Tornabene F (2018) Some novel numerical applications of Cosserat continua. Int J Comput Methods 15(06):1850054

38. Leonetti L, Fantuzzi N, Trovalusci P, Tornabene F (2019) Scale effects in orthotropic composite assemblies as micropolar continua: a comparison between weak-and strong-form finite element solutions. Materials 12(5):758

39. Fantuzzi N, Trovalusci P, Luciano R (2020) Material symmetries in homogenized hexagonal-shaped composites as Cosserat continua. Int $\mathrm{J}$ Multiscale Comput Eng 18(2):265-284

40. Fantuzzi N, Trovalusci P, Luciano R (2020) Multiscale analysis of anisotropic materials with hexagonal microstructure as micropolar continua. Int $\mathbf{J}$ Multiscale Comput Eng, 18(2)

41. Kouznetsova V, Geers MGD, Brekelmans WAM (2002) Multi-scale constitutive modelling of heterogeneous materials with a gradient-enhanced computational homogenization scheme. Int $\mathrm{J}$ Numerical Methods Eng 54(8):1235-1260

42. Kouznetsova VG, Geers MGD, Brekelmans WAM (2004) Multi-scale second-order computational homogenization of multi-phase materials: a nested finite element solution strategy. Comput Methods Appl Mech Eng 193(48-51):5525-5550

43. Peerlings RHJ, Fleck NA (2004) Computational evaluation of strain gradient elasticity constants. Int $\mathrm{J}$ Multiscale Comput Eng 2(4):599-619

44. Massart TJ, Peerlings RHJ, Geers MGD (2007) An enhanced multi-scale approach for masonry wall computations with localization of damage. Int J Numerical Methods Eng 69(5):1022-1059

45. Leismann T, Mahnken R (2015) Comparison of hyperelastic micromorphic, micropolar and microstrain continua. Int $\mathbf{J}$ Non-Linear Mech 77:115-127

46. Uzun B, Civalek Ö (2019) Nonlocal FEM formulation for vibration analysis of nanowires on elastic matrix with different materials. Math Comput Appl 24(2):38

47. Drugan WJ, Willis JR (1996) A micromechanics-based nonlocal constitutive equation and estimates of representative volume element size for elastic composites. J Mech Phys Solids 44(4):497-524

48. Luciano R, Willis JR (2000) Bounds on non-local effective relations for random composites loaded by configurationdependent body force. J Mech Phys Solids 48(9):1827-1849

49. Smyshlyaev VP, Cherednichenko KD (2000) On rigorous derivation of strain gradient effects in the overall behaviour of periodic heterogeneous media. J Mech Phys Solids 48(6-7):1325-1357

50. Assidi M, Ganghoffer JF (2012) Composites with auxetic inclusions showing both an auxetic behavior and enhancement of their mechanical properties. Composite Struct 94(8):2373-2382

51. Bacca M, Bigoni D, Dal Corso F, Veber D (2013) Mindlin second-gradient elastic properties from dilute two-phase Cauchy-elastic composites. Part i: Closed form expression for the effective higher-order constitutive tensor. Int J Solids and Struct 50(24):4010-4019

52. Barretta R, Luciano R, Marotti de Sciarra F (2015) A fully gradient model for Euler-Bernoulli nanobeams. Math Problems Eng 2015:1-8

53. Barretta R, Faghidian SA, Luciano R (2019) Longitudinal vibrations of nano-rods by stress-driven integral elasticity. Mech Adv Mater Struct 26(15):1307-1315

54. Ferreira AJM, Fantuzzi N (2020) MATLAB codes for finite element analysis 2nd edition: solids and structures. Springer, Netherlands

55. Eremeyev VA, Pietraszkiewicz W (2016) Material symmetry group and constitutive equations of micropolar anisotropic elastic solids. Math Mech Solids 21(2):210-221

56. Sokolowski M (1970) Theory of couple-stresses in bodies with constrained rotations, vol 26. Springer, Berlin

57. Scherphuis J (2019) Jaap's puzzle page. https://www. jaapsch.net/tilings/index.htm

58. Bigoni D, Noselli G (2010) Localized stress percolation through dry masonry walls. part I-Experiments. Euro J Mech-A/Solids 29(3):291-298

Publisher's Note Springer Nature remains neutral with regard to jurisdictional claims in published maps and institutional affiliations. 